\title{
Estimating the ballistic coefficient of a re-entry vehicle
}

\author{
P. Dodin, P. Minvielle and J.P. Le Cadre
}

\begin{abstract}
Theoretical bounds for estimating the ballistic coefficient of a ballistic object during the re-entry phase have been addressed. One essential characteristic of the vehicle trajectory is its deceleration when it reaches dense atmospheric layers. The intensity of the phenomenon is proportional to a scalar, called the ballistic coefficient. This leads to an highly nonlinear time-varying dynamic. To understand the dimensioning parameters for estimating the ballistic coefficient, accurate approximations of the Fisher information matrix are developed. The main result is a closed-form expression of a lower bound for the variance of the ballistic coefficient estimate.
\end{abstract}

\section{Notations}

- Let $S$ be the object cross-section, $C_{X}$ its drag coefficient and $M$ its mass,

- the ballistic coefficient $\beta$ is the product $C_{x} S / m$ and is expressed in $\mathrm{m}^{2} \mathrm{~kg}^{-1}$,

- $g o=-9.8 \mathrm{~ms}^{-2}$ is the gravitational acceleration (at ground level),

- alt is the altitude; alt $\triangleq y$ in the coordinate system of the Fig. 1,

- the atmospheric density $(\rho(y))$ is modelled by an exponential law: $\rho(y)=\rho_{0} \exp \left[-y / c_{\rho}\right]$ (in $\mathrm{kg} \mathrm{m}^{-3}$ ), with $c_{\rho}=7000 \mathrm{~m}$ and $\rho_{0}=1.2 \mathrm{~kg} \mathrm{~m}^{-3}$,

- $q(y) \triangleq \frac{1}{2} \rho(y) v^{2}$ is the dynamic pressure,

- $r$ denotes the target range and $v$ its velocity,

- FIM: Fisher information matrix; CRB: Cramér-Rao bound, RV: re-entry vehicle, LOS: line of sight,

- det: determinant; $A^{\mathrm{T}}: A$ transposed.

\section{Introduction}

Anti-ballistic defenses are confronted with the challenge of detecting, in a few seconds, swift non-cooperative targets with a view to locate them precisely and to allow interception. With anti-ballistic missile or anti-tactical-ballistic missile goals, those defenses use adapted sensors, such as the millimetre wave radar located at Kwajalein (Marshall Islands) [1], to track re-entry vehicles leaving a quiet exo-atmospheric phase to an endo-atmospheric phase with large aerodynamics loads and a sudden deceleration. The intensity of the phenomenon is proportional to a scalar, called the ballistic coefficient (denoted as $\beta$ for the sequel). The motion is obviously nonlinear and, furthermore, the dynamic is time-varying. However, it is especially important to estimate the ballistic coefficient of the target, since the radar needs to quantify the target deceleration so as to be able to extrapolate its position, to point it and

\footnotetext{
(C) The Institution of Engineering and Technology 2007

doi:10.1049/iet-rsn:20060064

Paper first received 12th May 2006 and in revised form 16th January 2007

P. Dodin and P. Minvielle are with the CEA CESTA, BP 233114 Le Barp, France

J.P. Le Cadre is with the IRISA/CNRS, Campus de Beaulieu, Rennes, France

E-mail: lecadre@irisa.fr
}

track it. Furthermore, it contributes to the classification and identification of the target (lethal target/decoy, type of debris etc.), by exploiting kinematic features. Note that the present problem setup largely differs from the tracking of an exo-atmospheric target [2], since it is precisely sudden change of atmospheric density which conditions the target motion.

The aim is to explore the ballistic coefficient estimability issue, that is to understand what makes it estimable and of how much. More specifically, our main concern is to develop accurate closed-form approximations of a lower bound of the variance of the ballistic coefficient estimate. Note that this paper does not address the question of how estimating the ballistic coefficient, meaning which procedure use. Many works have already been published on that subject, for example [1, 3, 4]. Besides, it is worth also stressing that the paper is far from classical calculations and that the complexity of the dynamic renders quite illusory a direct approach. Actually, the structure of the Fisher information matrix (FIM) is tremendously intricate. To overcome these difficulties, the following (simplifying) assumptions are made:

- the ballistic coefficient (denoted as $\beta$ ) is assumed to be constant throughout the whole scenario,

- idem for the re-entry angle (denoted as $\gamma$ ),

- the problem is restricted to a two-dimensional one.

It is worth also to stress that our approach is rather indirect. Instead coping with the FIM, we use intensively multilinear algebra so as to drastically reduce the calculation length [5]. Using this framework, difficulties reduce to obtain convenient closed-form approximations of the time derivative of the observation gradient vector.

Accuracy of this closed-form approximation of the Cramér-Rao lower bound (CRB) is investigated via a Monte-Carlo analysis [6]. To that aim, we refer to [7], where time-varying models of the ballistic coefficient are also considered for general three-dimensional re-entry scenarios. The problem is then considered in a tracking framework and it is shown that sequential Monte-Carlo methods perform quite satisfactorily. However, the aim, is whatever their impressive advantages, to determine the dimensioning factors for this tracking problem. 


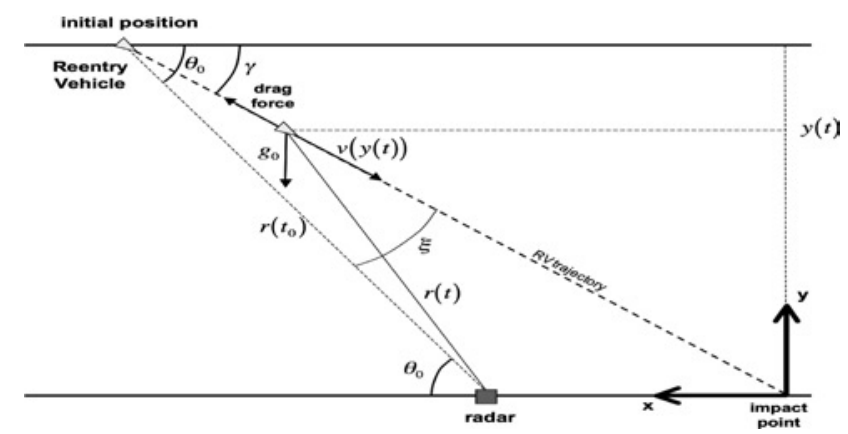

Fig. $12 D$ ballistic scenario: the problem parametrisation

This problem has a long and rich history [8], even if efforts have been mainly focused to date on the development of tracking methods $[3,9,10]$. For a long time, the workhorse has been the extended Kalman filter [3] and various versions [1] have been especially developed for this problem. Due to the highly nonlinear nature of the problem, sequential Monte-Carlo method is a strong challenger $[4,10]$ and allows to avoid uncertain linearisations, which is a definite advantage. Including a Markovian modelling (e.g. of the ballistic coefficient) leads to consider a Bayesian framework [8] and to replace the 'classical', CRB by the posterior Cramér-Rao bound (PCRB [11]). It is worth mentioning the works of Farina et al. for carrying out a systematic comparison between PCRB and the filter performance in this context $[6,12]$. However, as far as we know, there is no analytical explanation for investigating the estimability (improperly called observability) of the ballistic coefficient during the re-entry phase. More precisely, the main result is to show how and why it is the violent speed decrease due to the dynamic pressure which renders possible estimation (and tracking) of the ballistic coefficient during the re-entry phase.

To a large extent, this article is based on ballistic models developed by Allen et al. $[13,14]$. Note that Allen pushed the analysis far further than the elementary model used here and that, for time forward, this theory has been widely extended and refined [15]. Furthermore, the great ideas he developed are still the grounds of more recent developments.

The paper is organised as follows. The 2-D re-entry model is presented in Section 2, while Section 3 deals with methods for approximating the determinant (and sub-determinant) of the FIM. Using the results of Section 3 , it is then possible to obtain a closed form approximation of the CRB for the ballistic coefficient in case of range only measurements, which is the aim of Section 4. Following the guidelines of Section 3, this analysis is easily extended to range and bearing measurements (Section 5). In Section 6, simulation results are given. Detailed calculations are skipped in Appendices.

\section{2-D re-entry model}

In re-entry vehicle (RV) tracking, various dynamic models have been proposed for the endo-atmospheric phase $[1,3,4$, $10]$. Here, the article strictly focuses on the ballistic coefficient estimability and not on RV tracking. Since the full 3D problem is rather intricate, we shall restrict to a simpler but insightful 2D modelling. We choose the so-called Allen re-entry model described in $[13,16]$, commonly used in the field of re-entry aerodynamics. Close to [4], this 2D model is a zero-incidence endo-atmospheric model, with only aerodynamic drag and no lift. This strong hypothesis requires in particular that the RV is statically and dynamically stable, the possible incidence fluctuations have damped down, the ablation is limited and does not generate inertial and aerodynamic asymmetries, and so on. Dealing with high-speed objects with low $S C_{X} / M$, it is then correctly assumed in Allen model that the ballistic coefficient $\beta$ is constant. Furthermore, as we focus on the decelerating phase (typically under around $50-65 \mathrm{~km}$ altitude), it is possible to consider that the gravity becomes negligible compared to the drag force. With no gravity, there is no trajectory slope variation and the re-entry angle is constant. Subsequently, the re-entry angle $\gamma$ can be considered to be known. A reasonable assumption, taking into account prior observation before the re-entry phase and the fact that the lift force is non-significant.

Moreover, let us briefly mention other important assumptions: there is no centrifugal and Coriolis acceleration, the Earth is flat and does not rotate. Finally, note that the radar is assumed to be fixed. The parameters involved in the 2D modelling are described in Fig. 1.

Let $\gamma$ be the re-entry angle, and $v_{x}, v_{y}$ the two components of the target velocity vector, the state vector for this $2 \mathrm{D}$ re-entry problem is the four-dimensional vector

$$
\boldsymbol{x} \triangleq\left(r\left(t_{0}\right), v\left[y\left(t_{0}\right)\right], \theta\left(t_{0}\right), \beta\right)^{\mathrm{T}}
$$

The differential equation governing the system dynamic stands as follows

$$
\dot{v}_{y}=g_{0}+\sin (\gamma) \beta q(y) \quad \dot{v}_{x}=\cos (\gamma) \beta q(y)
$$

with

$$
q(y)=\frac{1}{2} \rho(y) v^{2}(y) \text { dynamic pressure }
$$

where $v$ is the norm of the velocity vector and $g_{0}$ is the gravity. As said previously, it is possible in the decelerating phase to consider that $g_{0}$ is negligible compared to dynamic pressure [13], then (2) is a bit simplified that is

$$
\dot{v}=\frac{1}{2} \beta \rho(y) v^{2}
$$

Note however that the simplicity of the above formulation is only apparent since the parameter $\rho$ is a function of the altitude $y$.

\subsection{Expression of the speed against altitude}

From (3) and considering the exponential atmospheric density model, Allen [13] was able to obtain an explicit solution of (3), yielding

$$
v(y)=v\left[y\left(t_{0}\right)\right] \exp \left(-\frac{1}{2 \sin (\gamma)} \beta c_{\rho} \rho(y)\right)
$$

Note that in (4), the velocity $v(y)$ is expressed as a function of the altitude $y$. It is possible to obtain an exact formula of the speed against time for example $v[y(t)]$ by substituting

$$
y(t)=\sin \left(\theta\left(t_{0}\right)\right) r\left(t_{0}\right)-\int_{t_{0}}^{t} v[y(\tau)] \sin (\gamma) \mathrm{d} \tau
$$

in (4). Then $\int_{t_{0}}^{t} v[y(\tau)] \mathrm{d} \tau$ is a solution of a differential equation. 


\subsection{Range approximation}

Our system is parameterised by two angles, namely $\gamma$ (the re-entry angle) and $\xi$ (the angle between the re-entry path and the initial line of sight $\theta_{t_{0}}$ ). The following range approximation is then instrumental in deriving closed-form FIM approximations.

Let us denote $v[y(t)]$ the speed along the re-entry path, and the angle $\zeta$ is used to have a negative cosine for the part of the speed vector which is along the initial line of sight, $\zeta \triangleq \pi-\xi$. The vector $v[y(t)]$ is then decomposed into two components: projection along the initial line of sight (associated with $\theta_{t_{t}}$ ), that is the radial component $v_{\mathrm{r}}(t)$, and the orthogonal (or angular) component $v_{\mathrm{a}}(t)$, that is

$$
v_{\mathrm{r}}(t)=v[y(t)] \cos (\zeta) \quad v_{\mathrm{a}}(t)=v[y(t)] \sin (\zeta)
$$

and, similarly

$$
\begin{array}{ll}
r_{\mathrm{r}}(t)=r[y(t)] \cos (\zeta) & r_{\mathrm{a}}(t)=r[y(t)] \sin (\zeta) \\
\dot{v}_{\mathrm{r}}(t)=\dot{v}[y(t)] \cos (\zeta) & \dot{v}_{\mathrm{a}}(t)=\dot{v}[y(t)] \sin (\zeta)
\end{array}
$$

A common assumption is that: $r_{\mathrm{a}}(t) \ll r_{\mathrm{r}}(t)$. Now, using the classic approximation $\sqrt{\left(A^{2}+B^{2}\right)} \simeq A+(1 / 2) B^{2} / A$, valid for real positive numbers such as $A \gg B$, the following range approximation is derived

$$
\begin{aligned}
r(t) & =\left(\left(r\left(t_{0}\right)+\int_{t_{0}}^{t} v_{\mathrm{r}} \mathrm{d} \tau\right)^{2}+\left(\int_{t_{0}}^{t} v_{\mathrm{a}} \mathrm{d} \tau\right)^{2}\right)^{1 / 2} \\
& \simeq r\left(t_{0}\right)+\int_{t_{0}}^{t} v_{\mathrm{r}} \mathrm{d} \tau+(1 / 2) \frac{\left(\int_{t_{0}}^{t} v_{\mathrm{a}} \mathrm{d} \tau\right)^{2}}{\left(r\left(t_{0}\right)+\int_{t_{0}}^{t} v_{\mathrm{r}} \mathrm{d} \tau\right)}
\end{aligned}
$$

This simple approximation will be of constant use subsequently.

\section{2D FIM and determinant approximations, for range-only measurements}

The object of this section is to investigate closed-form approximation of the FIM, so as to provide a closed-form expression of an accurate lower bound for the variance of any estimator of the ballistic coefficient $\beta$ (see Section 4). In this section, we restrict to range only measurements [17].

\subsection{FIM matrix}

Let $\boldsymbol{M}$ be the gradient vector of the observation along the target trajectory, that is

$$
\boldsymbol{M}_{t} \equiv\left(\frac{\partial r(t)}{\partial r\left(t_{0}\right)}, \frac{\partial r(t)}{\partial v\left[y\left(t_{0}\right)\right]}, \frac{\partial r(t)}{\partial \theta\left(t_{0}\right)}, \frac{\partial r(t)}{\partial \beta}\right)^{\mathrm{T}}
$$

and let $\boldsymbol{N}_{t}=\left(1 / \sigma_{r}\right) \boldsymbol{M}_{t}$, with $\sigma_{r}$ the range measurement standard deviation. In this deterministic context, the FIM calculation is quite standard and we have

$$
\mathbf{F I M}_{\left(t_{0}, t_{k}\right)}^{r}=\sum_{s=t_{0}}^{t_{k}} \boldsymbol{N}_{s} \boldsymbol{N}_{s}^{\mathrm{T}}=\boldsymbol{Z} \boldsymbol{Z}^{\mathrm{T}}
$$

with $\boldsymbol{Z}=\left[\boldsymbol{N}_{t_{0}}\left|\boldsymbol{N}_{t_{1}}\right| \cdots \mid \boldsymbol{N}_{t_{k}}\right]$ the matrix whose each column is the vector $\boldsymbol{N}_{t}$ from $t \stackrel{t_{k}}{=} t_{0}$ to $t=t_{k}$. It is well known that the determinant of the FIM is a convenient 'measure' of the system estimability. However, it is obvious that brute force calculations (even via symbolic computations) will lead to inextricable formulas due to the highly nonlinear nature of the system [see (6)], and will be of no help for understanding the key parameters governing the system estimability. Surprisingly, using basic multilinear algebra properties and some justified approximations, we shall show that it is possible to obtain relatively simple closed-form approximations of the FIM determinant and relevant sub-determinant.

\subsubsection{Cauchy-Binet formula and its consequences:} The Cauchy-Binet formula is instrumental for calculating the determinant $\operatorname{det}\left(\mathbf{F} \mathbf{I} \mathbf{M}_{\left.t_{0}, t_{k}\right)}^{r}\right)=\operatorname{det}\left(\boldsymbol{Z Z}^{\mathrm{T}}\right)$. More precisely, basic determinant properties (multilinear and alternate form) yield (see [5])

$$
\operatorname{det}\left(\mathbf{F I M}_{\left(t_{0}, t_{k}\right)}^{r}\right)=\sum_{0 \leq p \leq q \leq r \leq s \leq k}\left[\operatorname{det}\left(\boldsymbol{N}_{t_{p}}, \boldsymbol{N}_{t_{q}}, \boldsymbol{N}_{t_{r}}, \boldsymbol{N}_{t_{s}}\right)\right]^{2}
$$

Calculations are then reduced to calculations of elementary $4 \times 4$ matrices. Consider now a fourth-order expansion of the gradient vector $\left(\tilde{t}_{i} \triangleq t_{i}-t_{0}\right)$, that is,

$$
\boldsymbol{M}_{t_{i}}=\boldsymbol{M}_{t_{0}}+\tilde{t}_{i} \boldsymbol{M}_{t_{0}}^{(1)}+\frac{\tilde{t}_{i}^{2}}{2} \boldsymbol{M}_{t_{0}}^{(2)}+\frac{\tilde{t}_{i}^{3}}{6} \boldsymbol{M}_{t_{0}}^{(3)}
$$

and let us define the $4 \times 4$ square matrix

$$
\begin{aligned}
\boldsymbol{\Omega}(t) & \triangleq\left[\boldsymbol{M}, \frac{\mathrm{d} \boldsymbol{M}}{\mathrm{d} t}, \frac{\mathrm{d}^{2} \boldsymbol{M}}{\mathrm{d} t^{2}}, \frac{\mathrm{d}^{3} \boldsymbol{M}}{\mathrm{d} t^{3}}\right](t) \\
& \triangleq\left[\boldsymbol{M}_{t}, \boldsymbol{M}_{t}^{(1)}, \boldsymbol{M}_{t}^{(2)}, \boldsymbol{M}_{t}^{(3)}\right]
\end{aligned}
$$

Then, a very accurate approximation of the FIM determinant stands as follows

$$
\begin{aligned}
& \operatorname{det}\left(\mathbf{F I M}_{\left(t_{0}, t_{k}\right)}^{r}\right) \\
& =\frac{1}{\sigma_{r}^{8}} \sum_{0 \leq p<q<r<s \leq k}\left[\operatorname{det}\left(\boldsymbol{M}_{t_{p}}, \boldsymbol{M}_{t_{q}}, \boldsymbol{M}_{t_{r}}, \boldsymbol{M}_{t_{s}}\right)\right]^{2} \\
& \simeq R(k)\left[\operatorname{det}\left(\boldsymbol{\Omega}\left(t_{0}\right)\right)\right]^{2}
\end{aligned}
$$

with

$$
\begin{aligned}
& R(k)=\sum_{0 \leq p<q<r<s \leq k} Q^{2}\left(\tilde{t}_{p}, \tilde{t}_{q}, \tilde{t}_{r}, \tilde{t}_{s}\right) \quad \text { and } \\
& \operatorname{det}\left(\boldsymbol{\Omega}\left(t_{0}\right)\right)=\operatorname{det}\left(\boldsymbol{N}, \boldsymbol{N}^{(1)}, \boldsymbol{N}^{(2)}, \boldsymbol{N}^{(3)}\right)\left(t_{0}\right)
\end{aligned}
$$

In (13), $Q$ is the homogeneous $(p, q, r, s)$ polynomial

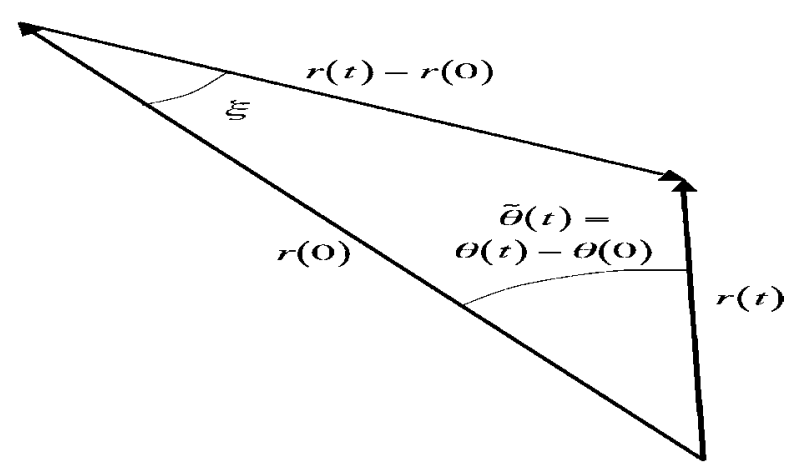

Fig. 2 Angular variation against range variation 
defined by $\left(t_{p}=t_{0}+p \delta, \delta\right.$ : inter-dwell time $)$

$$
\begin{aligned}
Q(p, q, r, s)= & \frac{1}{12}\left(q s^{2} r^{3}-q r^{2} s^{3}+q^{2} r s^{3}-q^{2} s r^{3}\right. \\
& +q^{3} s r^{2}-q^{3} s^{2} r+p r^{2} s^{3}-p s^{2} r^{3} \\
& +p q^{2} r^{3}-p q^{2} s^{3}+p q^{3} s^{2}-p q^{3} r^{2} \\
& +p^{2} s r^{3}-p^{2} s^{3} r+p^{2} q s^{3}-p^{2} q r^{3} \\
& +p^{2} q^{3} r-p^{2} q^{3} s+p^{3} s r^{2}-p^{3} s^{2} r \\
& \left.+p^{3} q s^{2}-p^{3} q r^{2}+q^{2} p^{3} s-q^{2} p^{3} r\right)
\end{aligned}
$$

Although this formula is already of great interest for our study, it still remains a problem. Indeed, the highly nonlinear dynamic is inducing a growing error in the Taylor approximation of formula (11), if all terms are computed based on the matrix $\operatorname{det}\left(\boldsymbol{M}, \mathrm{d} \boldsymbol{M} / \mathrm{d} t, \mathrm{~d}^{2} \boldsymbol{M} / \mathrm{d} t^{2}\right.$, $\left.\mathrm{d}^{3} \boldsymbol{M} / \mathrm{d} t^{3}\right)\left(t_{0}\right)$, at the initial instant $t_{0}$. Thus, we can refine previous approximations; this time evaluated in the vicinity of successive instants $t_{0}, \ldots, t_{k}$, yielding

$$
\operatorname{det}\left(\mathbf{F I M}_{\left(t_{0}, t_{k}\right)}^{r}\right) \simeq \frac{1}{\sigma_{r}^{8}} \sum_{0 \leq q \leq k} S(q, k)\left[\operatorname{det}\left(\boldsymbol{\Omega}\left(t_{q}\right)\right)\right]^{2}
$$

with

$$
S(q, k)=\sum_{p \leq q \leq r \leq s \leq k} Q^{2}\left(t_{p}-t_{q}, 0, t_{r}-t_{q}, t_{s}-t_{q}\right)
$$

The time period $t_{q}$ is chosen as the reference time for expansion, because of its intermediate position. Of course, $t_{r}$ could have been chosen instead. In Fig. 3, we show the expansion polynomials $S(i, 50)$ [see (14)] against $i$, whether $t_{q}$ or $t_{r}$ is chosen to develop the formula. Remark that they are not symmetric. A symmetric expansion can be obtained by computing the mean of the two former expansions. Not surprisingly, we shall see that best approximations are obtained via this symmetrised approximation.

It remains now to derive convenient closed-form approximations of the $\boldsymbol{\Omega}$ matrix.

\subsection{Closed-form approximations of the $\Omega$ matrix}

The calculation of a convenient closed-form approximation of the $\boldsymbol{\Omega}$ matrix will be obtained via some approximations. First, a crucial point is to obtain convenient approximations of the $\mathrm{d} \boldsymbol{M} / \mathrm{d} t$ vector.

Proposition 1: Using the system of exact equations (4) and (5), we have the following approximations of the speed partial derivatives as well as of its time derivatives (see

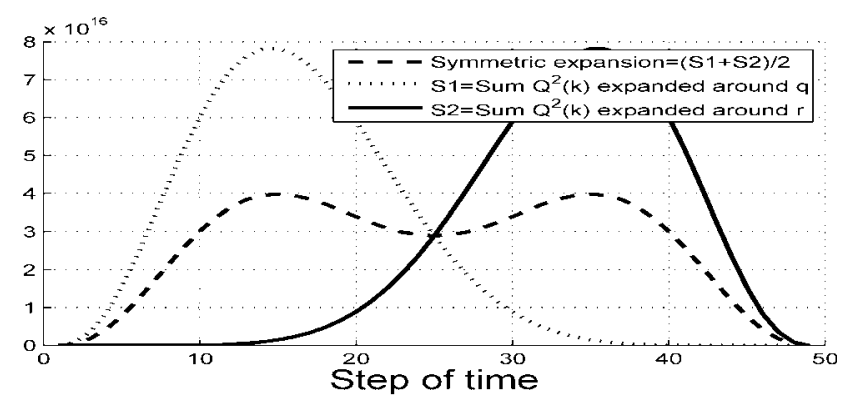

Fig. 3 Symmetric and unsymmetric expansions
Appendix A, for a proof)

$$
\begin{aligned}
\frac{\partial v[y(t)]}{\partial r\left(t_{0}\right)} & \simeq \epsilon(t) \frac{\sin \left(\theta\left(t_{0}\right)\right)}{\sin (\gamma)} \simeq 0 \\
\frac{\partial v[y(t)]}{\partial v\left[y\left(t_{0}\right)\right]} & \simeq \frac{v[y(t)]}{v\left[y\left(t_{0}\right)\right]} \\
\frac{\mathrm{d}}{\mathrm{d} t} \frac{\partial v[y](t)}{\partial r\left(t_{0}\right)} & \simeq \dot{\epsilon}(t) \frac{\sin \left(\theta\left(t_{0}\right)\right)}{\sin (\gamma)} \simeq 0 \\
\frac{\mathrm{d}}{\mathrm{d} t} \frac{\partial v[y](t)}{\partial v\left[y\left(t_{0}\right)\right]} & \simeq \epsilon(t) \simeq 0 \\
\frac{\mathrm{d}}{\mathrm{d} t} \frac{\partial v[y](t)}{\partial \beta} & \simeq \frac{1}{2} \rho(y(t)) v^{2} \\
\text { with } \epsilon(t) & \equiv \frac{1}{2} \beta \rho(y(t)) v[y(t)]
\end{aligned}
$$

These approximations are valid if $\epsilon(\mathrm{t})$ is small enough in regard to 1 , a reasonable hypothesis in the re-entry context. Indeed for our application, we typically have (cst: here denotes an integer, comprised between 1 and 5,) $v \simeq \operatorname{cst} 10^{3} \mathrm{~m} \mathrm{~s}^{-1}, \beta \simeq 10^{-4} \mathrm{~m}^{2} \mathrm{~kg}^{-1}$, and thus $\epsilon(t) \simeq$ $(\operatorname{cst} \rho(\mathrm{t})) / 20$.

To give the magnitude of $\rho(y)$ values, $\rho \simeq 1 \times$ $10^{-4} \mathrm{~kg} \mathrm{~m}^{-3}$ for $y=65 \mathrm{~km}, \rho \simeq 2 \times 10^{-3} \mathrm{~kg} \mathrm{~m}^{-3}$ for $y=45 \mathrm{~km}$ and $\rho \simeq 1.2 \mathrm{~kg} \mathrm{~m}^{-3}$ at ground level. We also suppose that $\gamma$ is sufficiently large enough for avoiding singularities.

Then, the following proposition summarises our approximations.

Proposition 2: Let us suppose valid the approximations of Proposition 1 and furthermore, assume that $r_{r}(t) \gg r_{a}(t)$. Additionally, let us denote $\tilde{\theta}(t)=\theta(t)-\theta\left(t_{0}\right)$, the angular change (sufficiently small, see Fig. 2). Then, the $\boldsymbol{\Omega}(t)$ matrix is a quasi-lower-triangular matrix, with only one non-zero coefficient above the diagonal $\left(\boldsymbol{\Omega}_{[3,4]}\right.$ namely) only one term, so that

1.

$$
\begin{aligned}
\operatorname{det}(\boldsymbol{\Omega}(t)) \simeq & \boldsymbol{\Omega}_{[1,1]}(t) \boldsymbol{\Omega}_{[2,2]}(t)\left[\boldsymbol{\Omega}_{[3,3]}(t) \boldsymbol{\Omega}_{[4,4]}(t)\right. \\
& \left.-\boldsymbol{\Omega}_{[4,3]}(t) \boldsymbol{\Omega}_{[3,4]}(t)\right]
\end{aligned}
$$

2. Using the above formula and convenient approximations of the $\boldsymbol{\Omega}_{[\mathrm{i}, \mathrm{j}]}$ coefficients, the following closed-form approximation of $\operatorname{det}(\boldsymbol{\Omega}(t))$ is finally obtained

$$
\begin{aligned}
\operatorname{det}(\boldsymbol{\Omega}(t)) \simeq & \frac{2 v_{r}}{v\left[y\left(t_{0}\right)\right]} \frac{\dot{v}^{2}}{\beta} \frac{r\left(t_{0}\right)}{r(t)} \cos ^{2}(\xi) \\
& \times\left(-\dot{\theta}+\{\tilde{\theta}(t)+\cot (\xi)\} \frac{\dot{r}(t)}{r(t)}\right)
\end{aligned}
$$

The proof is developed in Appendix B, (see Fig. 2 for the definition of the angle $\xi$ )

\section{Closed-form CRB approximation for the ballistic coefficient $\beta$ (range-only measurements)}

We shall now use the preceding results for deriving closedform approximations for the CRB related to the estimation of the ballistic coefficient $\beta$ in the range-only case. The main result of this section will be to emphasise that this 
lower bound is tightly related to the dynamic pressure. Thus, it is the abrupt deceleration induced by the increase of atmospheric density which renders it 'estimable'. A priori uncertainty is represented by a matrix $\boldsymbol{F}_{0}^{-1}=\operatorname{diag}$ $\left(\sigma_{r}^{2}, \sigma_{v}^{2}, \sigma_{\theta}^{2}, \sigma_{\beta}^{2}\right)$, so that

$$
\operatorname{FIM}_{\left(t_{k}\right)}^{r}=\boldsymbol{F}_{0}+\operatorname{FIM}_{\left(t_{1}, t_{k}\right)}^{r}
$$

The lower bound, $\mathbf{C R B}_{\beta}^{\mathbf{r}}$, for the coefficient $\beta$ is the $(4,4)$ term of $\left[\mathbf{F I M}_{\left(t_{k}\right)}^{r}\right]^{-1}$. Let $\boldsymbol{A}$ be the $(3 \times 3)$ matrix extracted from $\operatorname{FIM}_{\left(t_{k}\right)}^{r}$, excluding the last column and last line (the cofactor of $\left.\operatorname{FIM}_{\left(t_{k}\right)}^{r}(4,4)\right)$, then

$$
\boldsymbol{A}_{\left(t_{k}\right)}=\boldsymbol{A}_{0}+\boldsymbol{A}_{\left(t_{0}, t_{k}\right)}
$$

Now, using the classical formula yielding the inverse of a square matrix, we have

$$
\mathbf{C R B}_{\beta}^{\mathbf{r}}=\frac{1}{\operatorname{det}\left(\mathbf{F I M}_{\left(t_{k}\right)}^{r}\right)} \operatorname{det}\left(A\left(t_{k}\right)\right)
$$

Note that $\operatorname{det}\left(\boldsymbol{A}_{(t k)}\right)$ can be evaluated in the same way that the FIM determinant was evaluated. More precisely, let us define the $3 \times 3$ square matrix

$$
\boldsymbol{\Theta}(t) \equiv\left[\boldsymbol{M}, \frac{\mathrm{d} \boldsymbol{M}}{\mathrm{d} t}, \frac{\mathrm{d}^{2} \boldsymbol{M}}{\mathrm{d} t^{2}}\right](t)=\left[\boldsymbol{M}_{t}, \boldsymbol{M}_{t}^{(1)}, \boldsymbol{M}_{t}^{(2)}\right]
$$

then

$$
\operatorname{det}(\boldsymbol{\Theta}(t)) \simeq \prod_{i=1}^{3} \boldsymbol{\Omega}_{i, i} \simeq 2 \frac{v_{\mathrm{r}}}{v\left[y\left(t_{0}\right)\right]} \frac{r\left(t_{0}\right) \dot{v} \cos ^{2}(\xi)}{r(t) \sin (\xi)}
$$

More generally, using Lemma 2, we have

$$
\begin{aligned}
\operatorname{det}\left(\boldsymbol{A}_{\left(t_{0}, t_{k}\right)}\right)= & \frac{1}{\sigma_{r}^{6}} \sum_{0 \leq p \leq q \leq r \leq k} \operatorname{det}\left(\boldsymbol{M}_{t_{p}}, \boldsymbol{M}_{t_{q}}, \boldsymbol{M}_{t_{r}}\right)^{2} \\
= & \frac{1}{\sigma_{r}^{6}} \sum_{0 \leq q \leq k} \sum_{p \leq q \leq r \leq k} \operatorname{det}\left(\boldsymbol{M}_{t_{q}+\left(t_{p}-t_{q}\right)},\right. \\
& \left.\boldsymbol{M}_{t_{q}}, \boldsymbol{M}_{t_{q}+\left(t_{r}-t_{q}\right)}\right)^{2} \\
= & \frac{1}{\sigma_{r}^{6}} \sum_{0 \leq q \leq k} U(q, k) \cdot \operatorname{det}\left(\boldsymbol{\Theta}\left(t_{q}\right)\right)^{2} \\
\text { with } U(q, k)= & \sum_{p \leq q \leq r \leq k} P\left(t_{p}-t_{q}, 0, t_{r}-t_{q}\right)^{2}
\end{aligned}
$$

Here $P$ is the homogeneous polynomial

$$
P(p, q, r)=\frac{q r^{2}}{2}+\frac{p q^{2}}{2}+\frac{p^{2} r}{2}-\frac{q^{2} r}{2}-\frac{p r^{2}}{2}-\frac{p^{2} q}{2}
$$

(An evaluation of the polynomial function $U(q)$ can be found in Appendix C, Lemma 3.)

Collecting all the previous results and neglecting initial information about the $r, v, \theta$ parameters, we have the following result, which summarises the main contribution of this article.

Proposition 3: Let $\mathbf{C R B}_{\beta}^{\mathbf{r}}\left(t_{k}\right)$, the lower bound relative to the estimation of the ballistic coefficient $\beta$ at time $t_{k}$.
Then, the following closed-form approximation holds true

$$
\begin{aligned}
\mathbf{C R B}_{\beta}^{\mathbf{r}}\left(t_{k}\right) & \simeq \frac{\left(1 /\left(\sigma_{v}^{*} \sigma_{r}^{*} \sigma_{\theta}\right)^{2}\right)+f(k)}{1 /\left(\sigma_{v}^{*} \sigma_{r}^{*} \sigma_{\theta} \sigma_{\beta}\right)^{2}+\left(1 / \sigma_{\beta}^{2}\right) f(k)+g(k)} \\
& =\left(\frac{1}{\sigma_{\beta}^{2}}+\frac{g(k)}{\left(1 /\left(\sigma_{v}^{*} \sigma_{r}^{*} \sigma_{\theta}\right)^{2}\right)+f(k)}\right)^{-1} \\
& \simeq\left(\frac{1}{\sigma_{\beta}^{2}}+\frac{g(k)}{f(k)}\right)^{-1}
\end{aligned}
$$

with

$$
\begin{aligned}
f(k)= & \frac{1}{\sigma_{r}^{6}} \sum_{0 \leq q \leq k} U(q, k)\left[\frac{v_{\mathrm{r}}}{v\left[y\left(t_{0}\right)\right]} \frac{2 r\left(t_{0}\right) \dot{v} \cos ^{2}(\xi)}{r(t) \sin (\xi)}\right]^{2} \\
g(k)= & \frac{1}{\sigma_{r}^{8}} \sum_{0 \leq q \leq k} S(q, k)\left[\frac{v_{\mathrm{r}}}{v\left[y\left(t_{0}\right)\right]} \frac{2 \dot{v}^{2} r\left(t_{0}\right)}{\beta r(t)} \cos ^{2}(\xi)\right. \\
& \left.\times\left(-\dot{\theta}+(\tilde{\theta}(t)+\cot (\xi)) \frac{r(\dot{t})}{r(t)}\right)\right]^{2}
\end{aligned}
$$

This approximation is initially equal to $\sigma_{\beta}^{2}$ and brutally decreases as soon as the quotient $\dot{v} / \beta=(1 / 2) p(y) . v^{2}$ becomes sufficiently high that it is impossible to neglect its effect. Moreover, considering the functions $f$ and $g$, we see that the effect of the term $\dot{v} / \beta$ is amplified by a polynomial function (in $k$ ), whose greater exponent is 8 (i.e. it is $\left.\propto k^{8}\right)$. Numerical experiments show that the effect of dynamic pressure for estimating $\beta$ becomes perceptible at an altitude of $65 \mathrm{~km}$, where $\rho(y) \cdot v^{2}=$ cst $\times 100$ if $v=\mathrm{cst} \times 10^{3} \mathrm{~m} \mathrm{~s}^{-1}$. We also remark that when the angle $\xi$ tends to $\pi / 2$, the functions $f(k)$ and $g(k)$ tend to zero, then become constant functions.

We can extract from our approximation additional information. Indeed, if we locate at the points where the time derivative of the CRB is zero; then at this point we have actually

$$
\mathbf{C R B}^{\prime}=-\frac{(g / f)^{\prime}}{\left(\sigma_{\beta}^{2}+g(k) / f(k)\right)^{2}} \quad \text { and if } \mathbf{C R B}^{\prime}=0
$$

we have $(g / f)^{\prime}=0=$ or $g^{\prime} / f^{\prime}=g / f$

$$
\mathbf{C R B}=\left(\frac{1}{\sigma_{\beta}^{2}}+\frac{g^{\prime}(k)}{f^{\prime}(k)}\right)^{-1}
$$

To obtain the derivatives $f^{\prime}(k)$ and $g^{\prime}(k)$ we just have to remark that these functions are discrete sums indexed on $k$ (exactly like integrals approximations), hence the derivative is nothing else than the last term of the sum. In our case, elementary calculations yield

$$
\begin{aligned}
& \mathbf{C R B}_{t_{k} / \mathbf{C R B}^{\prime}=0}^{r}=\left[\frac{1}{\sigma_{\beta}^{2}}+\frac{1}{\sigma_{r}^{2}} \frac{S(k, k)}{U(k, k)}\left[\frac{q(y)}{\beta}\right.\right. \\
& \left.\left.\times\left(\sin ^{2}(\xi)\left(-\dot{\theta}+\tilde{\theta}\left(t_{k}\right) \frac{\dot{r}\left(t_{k}\right)}{r\left(t_{k}\right)}\right)+\cos ^{2}(\xi) \frac{\dot{r}\left(t_{k}\right)}{r\left(t_{k}\right)}\right)\right]^{2}\right]^{-1}
\end{aligned}
$$

Thus, the estimability of the parameter $\beta$ is proportional to the product of the dynamic pressure by the relative range derivative if there is no orthogonal part of the dynamic, typically no lift, and by the angular derivative if there is non-radial part. 


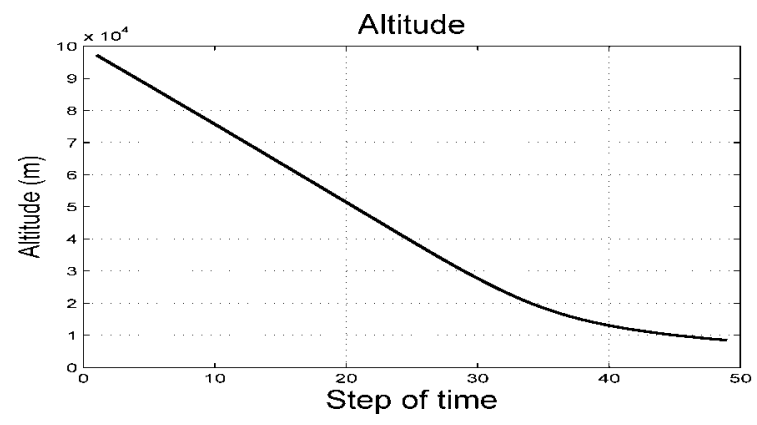

Fig. 4 RV altitude evolution

\section{Computation of the CRB of coefficient $\beta$ with range and bearing measurement}

Up to now, we restricted to range-only measurements. Considering range and bearing measurements requires only a slight extension of preceding derivations. More precisely, using an approximated chain rule that is $(\alpha$ : generic parameter):

$$
\frac{\partial \theta(t)}{\partial \alpha} \simeq \frac{\partial \theta(t)}{\partial r(t)} \frac{\partial r(t)}{\partial \alpha}=\frac{\tan (\xi)}{r(t)} \frac{\partial r(t)}{\partial \alpha}
$$

the bearings-only FIM (denoted as $\operatorname{FIM}_{\left(t_{0}, t_{k}\right)}^{\theta}$ is straightforwardly obtained

$$
\mathbf{F I M}_{\left(t_{0}, t_{k}\right)}^{\theta}=\sum_{s=t_{0}}^{t_{k}} \frac{\tan ^{2}(\xi)}{r(t)^{2} \sigma_{\theta}^{2}} \mathbf{M}_{s} \mathbf{M}_{s}^{T}
$$

Assuming that the analysis duration is not too important, we have

$$
\begin{aligned}
\mathbf{F I M}_{\left(t_{0}, t_{k}\right)}^{\theta, r} & \equiv \mathbf{F I M}_{\left(t_{0}, t_{k}\right)}^{\theta}+\mathbf{F I M}_{\left(t_{0}, t_{k}\right)}^{r} \\
& \simeq\left(\frac{\tan ^{2}(\xi)}{r\left(t_{0}\right)^{2} \sigma_{\theta}^{2}}+\frac{1}{\sigma_{r}^{2}}\right) \sum_{s=t_{0}}^{t_{k}} \mathbf{M}_{s} \mathbf{M}_{s}^{\mathrm{I}}
\end{aligned}
$$

\begin{tabular}{|c|c|c|}
\hline Name & Description & Value \\
\hline$[x, y]$ & $\begin{array}{l}\text { target initial } \\
\text { coordinates }\end{array}$ & [24 $6897 \mathrm{~m}, 95921 \mathrm{~m}]$ \\
\hline$\left[v_{x}, v_{y}\right]$ & $\begin{array}{l}\text { target initial speed } \\
\text { vector }\end{array}$ & {$\left[-6278 \mathrm{~m} \mathrm{~s}^{-1},-2100 \mathrm{~m} \mathrm{~s}^{-1}\right]$} \\
\hline$\beta$ & ballistic coefficient & $5 \times 10^{-4} \mathrm{~m}^{2} \mathrm{~kg}^{-1}$ \\
\hline$\xi$ & $\begin{array}{l}\text { angle between initial } \\
\text { LOS and speed vector }\end{array}$ & $0.02 \mathrm{rad}$ \\
\hline$\left[\sigma_{r}, \sigma_{\theta}\right]$ & measurement errors std & {$\left[5 \mathrm{~m}, 1 \times 10^{-4} \mathrm{rad}\right]$} \\
\hline $\mathrm{dt}$ & $\begin{array}{l}\text { time interval between } \\
\text { measurements }\end{array}$ & $1 \mathrm{~s}$ \\
\hline$\gamma$ & re-entry angle & $0.323 \mathrm{rad}$ \\
\hline
\end{tabular}

Thus, (27) is just replaced by

$$
\mathbf{C R B}_{\beta}^{\theta, \mathbf{r}}\left(t_{k}\right) \simeq\left(\frac{1}{\sigma_{\beta}^{2}}+\frac{i(k)}{h(k)}\right)^{-1}
$$

Table : Scenario parameters

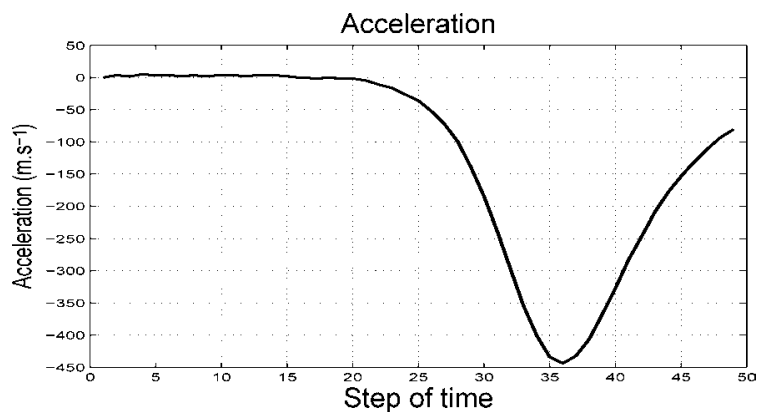

Fig. $5 R V$ deceleration evolution

$$
\begin{aligned}
& \text { with } \\
& \begin{aligned}
h(k)= & s(r, \theta)^{6} \sum_{0 \leq q \leq k} U(q, k)\left(\frac{v_{\mathrm{r}}}{v\left[y\left(t_{0}\right)\right]} \frac{2 r\left(t_{0}\right) \dot{v} \cos ^{2}(\xi)}{r(t) \sin (\xi)}\right)^{2} \\
i(k)= & s(r, \theta)^{8} \sum_{0 \leq q \leq k} S(q, k) \cdot\left[\frac{v_{\mathrm{r}}}{v\left[y\left(t_{0}\right)\right]} \frac{2 \dot{v}^{2} r\left(t_{0}\right)}{\beta r(t)} \cos ^{2}(\xi)\right. \\
& \left.\times\left(-\dot{\theta}+(\tilde{\theta}(t)+\cot (\xi)) \frac{\dot{r}(t)}{r(t)}\right)\right]^{2} \\
s(r, \theta)= & \sqrt{\frac{\tan ^{2}(\xi)}{r\left(t_{0}\right)^{2} \sigma_{\theta}^{2}}+\frac{1}{\sigma_{r}^{2}}}
\end{aligned}
\end{aligned}
$$

The contribution of the bearing measurement in the FIM exists if $\sin (\xi)$ is strictly positive, which means an angular relative displacement. Note that it is also proportional to the inverse of the range. Quite similarly to the range only case, we obtain the very simple formula for the CRB, at critical instants

$$
\begin{aligned}
& \mathbf{C R B}_{t_{k} / \mathbf{C R B}^{\prime}=0}^{r, \theta}=\left[\frac{1}{\sigma_{\beta}^{2}}+s(r, \theta)^{2} \frac{S(k, k)}{U(k, k)}\right. \\
& \times\left[\frac{\dot{v}}{\beta}\left(\sin ^{2}(\xi)\left(-\dot{\theta}+\tilde{\theta}\left(t_{k}\right) \frac{\dot{r}\left(t_{k}\right)}{r\left(t_{k}\right)}+\cos ^{2}(\xi) \frac{\dot{r}\left(t_{k}\right)}{r\left(t_{k}\right)}\right)\right]^{2}\right]^{-1}
\end{aligned}
$$

\section{Simulation results for range and bearing measurements}

We present results for range and bearing measurements in order to show the approximation validity and to illustrate its use. The studied scenario is described in Table 1:

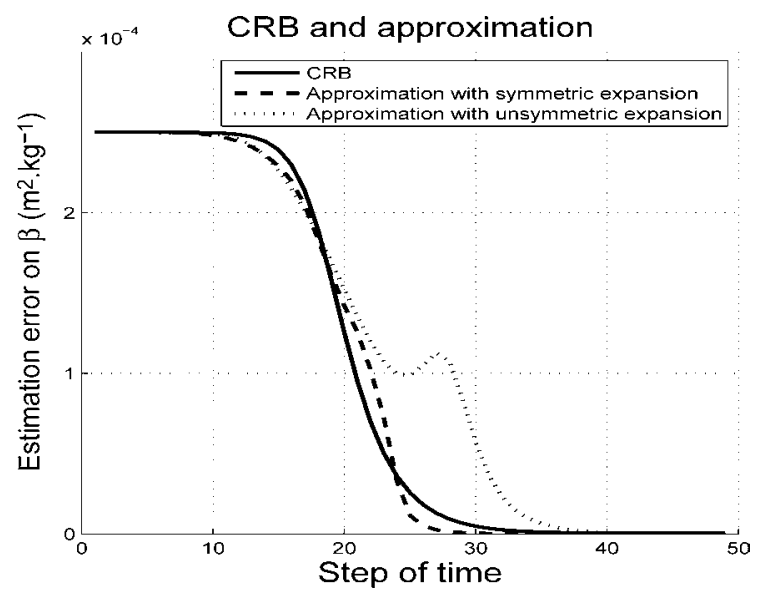

Fig. $6 \beta$ estimation error bounds $\left(\sigma_{\beta}=2.5 \times 10^{-4} \mathrm{~m}^{2} \mathrm{~kg}^{-1}\right)$ 


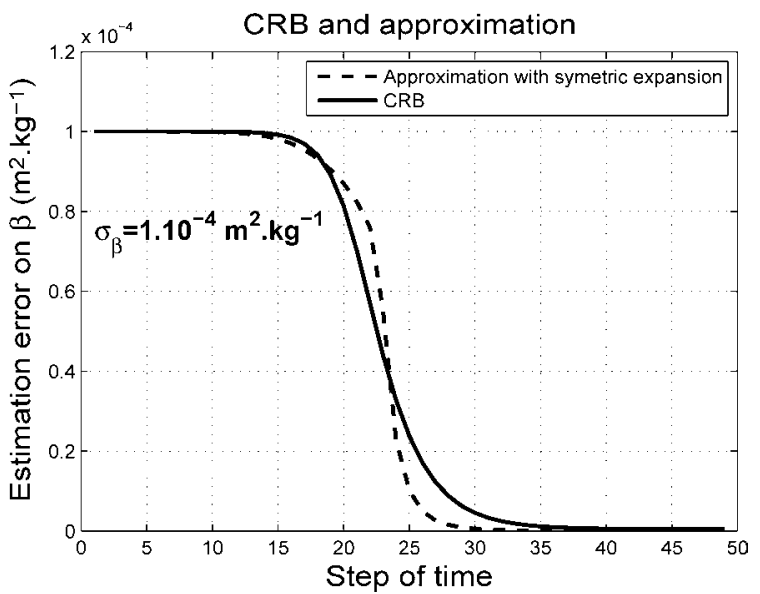

Fig. $7 \beta$ estimation error bounds $\left(\sigma_{\beta}=10^{-4} \mathrm{~m}^{2} \mathrm{~kg}^{-1}\right)$

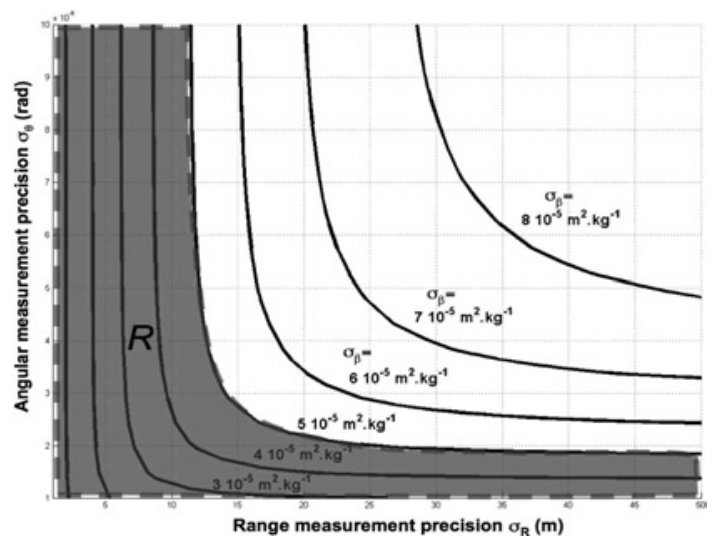

Fig. 8 Requirements on radar measurement errors

In Figs. 4 and 5, the RV trajectory altitude and deceleration are, respectively, represented during the $50 \mathrm{~s}$ radar observation. We shall note here that the altitude $65 \mathrm{~km}$ is related to the 15 -th measurement period.

In Figs. 6 and 7, we show the $\beta$ estimation error bounds provided by the exact $\mathrm{CRB}$ and by the approximation. $\sigma_{\beta}$ is set, respectively, to $2.5 \times 10^{-4} \mathrm{~m}^{2} \mathrm{~kg}^{-1}$ and $10^{-4} \mathrm{~m}^{2} \mathrm{~kg}^{-1}$. The continuous line stands for the exact value of $\sqrt{\mathbf{C R B}_{\beta}}$, whereas the dashed line represents the approximation with the symmetric expansion described in Section 3. On Fig. 6, the approximation obtained with the unsymmetric expansion is represented in dotted line. In both cases, the CRB curve is at first flat. This means that the measurements do not yet give any information that could lead to a decrease of the initial estimation error $\sigma_{\beta}$. Then a strong decrease begins at time 15 when the dynamic pressure effect becomes noticeable (roughly at $65 \mathrm{~km}$ altitude). At this moment, the deceleration $\dot{v}$ enhances the value of the FIM determinant and leads the CRB value to zero. Note that nearly at the same moment the approximated and exact bounds indicate that the $\beta$ coefficient starts to be observable.

Concerning the computation requirement, the approximation is not demanding. Once the target trajectory simulated and the $U$ and $S$ polynomials computed, the result is computed quasi-instantaneously. Compared to the exact $\mathrm{CRB}$, its computation is several decades faster.

Fig. 8 illustrates a basic application to radar requirements. Assuming an initial $\sigma_{\beta}$ value of $10^{-4} \mathrm{~m}^{2} \mathrm{~kg}^{-1}$ and a radar rate of $1 \mathrm{~Hz}$, it represents at $40 \mathrm{~km}$ altitude the approximated bound in function of the range and angle measurement errors. Then, if one wishes there a ballistic coefficient precision of $5 \times 10^{-5} \mathrm{~m}^{2} \mathrm{~kg}^{-1}$, it is straightforward to obtain the $R$ requirement area in radar measurement errors. Note that the approximation precision is sufficient enough in this parametric analysis.

In the same way, it is possible to make use of the closedform approximation (32) to evaluate dynamically at a given time what are the radar resources (scan rate, measurement errors etc.) which must be allocated to the tracked targets to obtain a given $\sigma_{\beta}$ estimation precision at a certain altitude.

\section{Conclusion}

Analysing the estimability of the ballistic coefficient during the re-entry phase is a challenging problem. A realistic highly nonlinear model of the target trajectory, based on Allen re-entry works, has been considered. The main results are closed-form approximations of the CRB. Simulation experiments show us that they are quite accurate. These novel results enhance the potential to understand the ballistic coefficient estimability. They can be quite useful for extracting dimensioning factors that determine ballistic coefficient estimation. For instance, they can provide a way to determine radar requirements or to improve the sensor management.

Some future work could consist in evaluating the ballistic coefficient estimability in more general situations. For example, it would be useful to extend the current results to the case where the radar is not in the trajectory plane, where the re-entry angle gamma is not perfectly known or where the ballistic coefficient varies.

\section{References}

1 Cardillo, G.P., Mrstick, A.V., and Plambeck, T.: 'A track filter for reentry objects with uncertain drag', IEEE Trans. Aerosp. Electron. Syst., 1999, 35, (2), pp. 394-409

2 Yeddanapudi, M., Shalom, Y.B., Pattipati, K.R., and Deb, S.: 'Ballistic missile track initiation from satellite observations', IEEE Trans. Aerosp. Electron. Syst., 1995, 31, (3), pp. 1054-1069

3 Costa, P.J., and Moore, W.H.: 'Extended Kalman-Bucy filters for radar tracking and identification'. Proc. IEEE National Radar Conf., 1991, pp. 127-131

4 Farina, A., Ristic, B., and Benvenuti, D.: 'Tracking a ballistic target, comparison of several nonlinear filters', IEEE Trans. Aerosp. Electron. Syst., 2002, 38, (3), pp. 854-867

5 Le Cadre, J.-P.: 'Properties of estimability criteria for target motion analysis', IEE Proc., Radar Sonar Navig., 1998, 145, (2), pp. 92-99

6 Ristic, B., Farina, A., Benvenuti, D., and Arulampalam, S.: 'Performance bounds and comparison of nonlinear filters for tracking a ballistic object on re-entry', IEE Proc., Radar Sonar Navig., 2003, 150, (2), pp. 65-70

7 Dodin, P., Minvielle, P., and Le Cadre, J.-P.: 'Re-entry vehicle tracking, observability and theoretical bound'. Proc. 8th Int. Conf. Inf. Fusion, PA, USA, July 2005, pp. 197-204

8 Minvielle, P.: 'Decades of improvement in re-entry ballistic vehicle tracking', IEEE Aerosp. Electron. Syst. Mag., 2005, 20, (8), pp. 1-14

9 Siouris, G., Cheng, G., and Wang, J.: 'Tracking an incoming missile using an extended interval kalman filter', IEEE Trans. Aerosp. Electron. Syst., 1997, 33, (1), pp. 232-240

10 Minvielle, P.: 'Tracking a ballistic re-entry vehicle with a sequential monte-carlo filter'. Proc. IEEE Aerospace Conf., Big Sky, USA, March 2002, pp. 1773-1787

11 Tichavsky, P., Muravchik, C.H., and Nehorai, A.: 'Posterior Cramer-Rao bounds for disrete-time nonlinear filtering', IEEE Trans. Signal Process., 1998, 46, (5), pp. 1386-1396

12 Farina, A., Ristic, B., and Timoneri, L.: 'Cramer-Rao bound for nonlinear filtering with $P_{d}<1$ and its applications to target tracking', IEEE Trans. Signal Process., 2002, 50, (8), pp. 1916-1924

13 Allen, H.J., and Eggers, A.J.: 'A study of the motion and aerodynamic heating of ballistic missiles entering the earth's atmosphere at high supersonic speeds'. NACA Technical note 1381, 1958

14 Allen, H.J., and Tobak, M.: 'Dynamic stability of vehicles ascending or descending paths through the atmosphere' (NACA'Technical note 4275'July 1958) 
15 Allen, H.J.: 'Motion of a ballistic missile angularly misaligned with the flight path upon entering the atmosphere and its effect upon aerodynamic heating, aerodynamic loads, and miss distance'. NACA Technical note 4048, October 1957

16 Galais, P.: 'Mécanique du vol. CEA/CESTA Technical report', 1998

17 Ristic, B., Arulampalam, S., and Carthy, Mc: 'Target motion analysis using range-only measurements: algorithms, performance and application to INGARA ISAR Data'. DSTO Technical Report TR-1095, January 2001

\section{Appendix A: partial derivative approximations}

We begin this rather technical appendix by proving the validity of the approximations given in Proposition 1 [(15)-(19)], which are instrumental for calculating closedform approximations of the CRB.

\subsection{Validity of approximations (15) and (16)}

First, let us recall the exact dynamic system equations

$$
\begin{aligned}
v[y(t)] & =v\left(y\left(t_{0}\right)\right) \exp \left(-\frac{1}{2 \sin (\gamma)} \beta c_{\rho} \rho(y(t))\right) \\
y(t) & =\sin \left(\theta\left(t_{0}\right)\right) r\left(t_{0}\right)-\int_{t_{0}}^{t} v[y(\tau)] \sin (\gamma) \mathrm{d} \tau
\end{aligned}
$$

Differentiating the first row of (35), we obtain

$$
\begin{aligned}
\frac{\partial v[y(t)]}{\partial r\left(t_{0}\right)} & =\frac{\rho(t) \beta v[y(t)]}{2 \sin (\gamma)} \frac{\partial y(t)}{\partial r\left(t_{0}\right)}=\frac{\epsilon(t)}{\sin (\gamma)} \frac{\partial y(t)}{\partial r\left(t_{0}\right)} \\
\frac{\partial v[y(t)]}{\partial v\left[y\left(t_{0}\right)\right]} & =\frac{\rho(t) \beta v[y(t)]}{2 \sin (\gamma)} \frac{\partial y(t)}{\partial v\left[y\left(t_{0}\right)\right]}+\frac{v[y(t)]}{v\left[y\left(t_{0}\right)\right]} \\
& =\frac{\epsilon(t)}{\sin (\gamma)} \frac{\partial y(t)}{\partial v\left[y\left(t_{0}\right)\right]}+\frac{v[y(t)]}{v\left[y\left(t_{0}\right)\right]}
\end{aligned}
$$

Differentiating the second row of (35), we obtain

$$
\begin{aligned}
\frac{\partial y(t)}{\partial r\left(t_{0}\right)} & =\sin \left(\theta\left(t_{0}\right)\right)-\int_{t_{0}}^{t} \frac{\partial v[y(\tau)]}{\partial r\left(t_{0}\right)} \sin (\gamma) \mathrm{d} \tau \\
\frac{\partial y(t)}{\partial v\left[y\left(t_{0}\right)\right]} & =-\int_{t_{0}}^{t} \frac{\partial v[y(\tau)]}{\partial v\left[y\left(t_{0}\right)\right]} \sin (\gamma) \mathrm{d} \tau
\end{aligned}
$$

If we substitute (37) (first row) in (36) (first row) and (37) (second row) in (36) (second row), we obtain

$$
\begin{aligned}
& \frac{\partial v[y(t)]}{\partial r\left(t_{0}\right)}=\frac{\epsilon(t)}{\sin (\gamma)} \sin \left(\theta\left(t_{0}\right)\right)-\frac{\epsilon(t)}{\sin (\gamma)} \int_{t_{0}}^{t} \frac{\partial v[y(\tau)]}{\partial r\left(t_{0}\right)} \sin (\gamma) \mathrm{d} \tau \\
& \frac{\partial v[y(t)]}{\partial v\left[y\left(t_{0}\right)\right]}=-\frac{\epsilon(t)}{\sin (\gamma)} \int_{t_{0}}^{t} \frac{\partial v[y(\tau)]}{\partial v\left[y\left(t_{0}\right)\right]} \sin (\gamma) \mathrm{d} \tau+\frac{v[y(t)]}{v\left[y\left(t_{0}\right)\right]}
\end{aligned}
$$

To derive convenient approximations of the partial derivatives $\partial v[y(\tau)] / \partial r\left[y\left(t_{0}\right)\right]$ and $\partial v[y(t)] / \partial v\left[y\left(t_{0}\right)\right]$ we define first the $S$ and $T$ integrals

$$
S=\int_{t_{0}}^{t} \frac{\partial v[y(\tau)]}{\partial r\left(t_{0}\right)} \mathrm{d} \tau, \quad T=\int_{t_{0}}^{t} \frac{\partial v[y(\tau)]}{\partial v\left[y\left(t_{0}\right)\right]} \mathrm{d} \tau
$$

Defining $\dot{S} \triangleq \mathrm{d} S / \mathrm{d} t$ and $T \triangleq \mathrm{d} T / \mathrm{d} t, \dot{S}$ and $\dot{T}$ are solutions of the following differential system

$$
\begin{aligned}
& \frac{\partial v[y(t)]}{\partial r\left[y\left(t_{0}\right)\right]}=\dot{S}=\frac{\epsilon(t)}{\sin (\gamma)} \sin \left(\theta\left(t_{0}\right)\right)-\epsilon(t) S \\
& \frac{\partial v[y(t)]}{\partial v\left[y\left(t_{0}\right)\right]}=\dot{T}=-\epsilon(t) T+\frac{v[y(t)]}{v\left[y\left(t_{0}\right)\right]} \\
& S\left(t_{0}\right)=0 \quad T\left(t_{0}\right)=0
\end{aligned}
$$

This can be solved to give (just differentiate to verify)

$$
\begin{aligned}
S= & \exp \left[-\int_{t_{0}}^{t} \epsilon(s) \mathrm{d} s\right] \int_{t_{0}}^{t} \exp \left[\int_{t_{0}}^{s} \epsilon(w) \mathrm{d} w\right] \\
& \times \frac{\epsilon(s)}{\sin (\gamma)} \sin \left(\theta\left(t_{0}\right)\right) \mathrm{d} s \\
T= & \exp \left[-\int_{t_{0}}^{t} \epsilon(s) \mathrm{d} s\right] \int_{t_{0}}^{t} \exp \left[\int_{t_{0}}^{s} \epsilon(w) \mathrm{d} w\right] \frac{v[y(s)]}{v\left[y\left(t_{0}\right)\right]} \mathrm{d} s
\end{aligned}
$$

Substituting (41) in (40), the following majorations are straightforwardly deduced

$$
\begin{aligned}
& \left|\frac{\partial v[y(t)]}{\partial r\left(t_{0}\right)}\right|=|\dot{S}| \leq \frac{\epsilon(t) \sin \left(\theta\left(t_{0}\right)\right)}{\sin (\gamma)}+\epsilon(t) \int_{t_{0}}^{t} \frac{\epsilon(s) \sin \left(\theta\left(t_{0}\right)\right)}{\sin (\gamma)} \mathrm{d} s \\
& \left|\frac{\partial v[y(t)]}{\partial v\left[y\left(t_{0}\right)\right]}\right|=|\dot{T}| \leq \frac{v[y(t)]}{v\left[y\left(t_{0}\right)\right]}+\epsilon(t) \int_{t_{0}}^{t} \frac{v[y(s)]}{v\left[y\left(t_{0}\right)\right]} \mathrm{d} s
\end{aligned}
$$

This proves formulas (15) and (16).

b) Equations (17) and (18)

To prove formula (17), and (18), we note that $\dot{v}=\frac{1}{2} \rho(t) \beta v^{2}$ so that straightforward calculations yield

$$
\begin{gathered}
\frac{\mathrm{d}}{\mathrm{d} t} \frac{\partial v[y(t)]}{\partial r\left(t_{0}\right)}=\frac{\partial \dot{v}[y(t)]}{\partial r\left(t_{0}\right)}=2 \epsilon(t) \frac{\partial v[y(t)]}{\partial r\left(t_{0}\right)} \\
\frac{\mathrm{d}}{\mathrm{d} t} \frac{\partial v[y(t)]}{\partial v\left[y\left(t_{0}\right)\right]}=\frac{\partial \dot{v}[y(t)]}{\partial v\left[y\left(t_{0}\right)\right]}=2 \epsilon(t) \frac{\partial v[y(t)]}{\partial v\left[y\left(t_{0}\right)\right]}
\end{gathered}
$$

Then, using the approximations of (15) and (16), approximations 17 and 18 are straightforwardly deduced.

c) Equations (19)

Thanks to (35), we obtain

$$
\frac{\partial v}{\partial \beta}=-\left(\frac{1}{2 \sin \gamma} \rho c_{\rho}\right) v
$$

whence

$$
\frac{\mathrm{d}}{\mathrm{d} t} \frac{\partial v}{\partial \beta}=\frac{\partial}{\partial \beta}\left(\frac{1}{2} \beta \rho v^{2}\right)=\frac{1}{2} \rho v^{2}\left(1-2 \epsilon \frac{\rho c_{\rho}}{v \sin (\gamma)}\right)
$$

But $c_{\rho}$ and $v$ are of the same order. For instance, we have $c_{\rho}=7000$ and $v=\mathrm{cst} \times 1000 \mathrm{~m} \mathrm{~s}^{-1}$. This proves formula (19).

Now, we have to calculate the components of the gradient vectors $\boldsymbol{M}_{t}, \boldsymbol{M}_{t}^{(1)}, \boldsymbol{M}_{t}^{(2)}, \boldsymbol{M}_{t}^{(3)}$. To that aim, the following elementary lemma is also instrumental:

Lemma 1: Let $p$ be a real parameter, like $r\left(t_{0}\right), v\left[y\left(t_{0}\right)\right]$ or $\beta$, and consider the following range approximation

$$
r=X+\frac{1}{2} \frac{Y^{2}}{X} \text { with } X=r\left(t_{0}\right) \int_{t_{0}}^{t} v_{\mathrm{r}} \mathrm{d} \tau, \quad Y=\int_{t_{0}}^{t} \nu_{\mathrm{a}} \mathrm{d} \tau
$$




$$
\frac{\partial r}{\partial p}=\frac{\partial X}{\partial p}+\frac{\partial Y}{\partial p} \frac{Y}{X}-\frac{1}{2}\left(\frac{Y}{X}\right)^{2} \frac{\partial X}{\partial p}
$$

and

$$
\begin{aligned}
\frac{\mathrm{d}}{\mathrm{d} t} \frac{\partial r}{\partial p}= & \frac{\partial v_{r}}{\partial p}+\frac{\partial Y}{\partial p} \frac{\mathrm{d}\left(\frac{Y}{X}\right)}{\mathrm{d} t}+\frac{Y}{X} \frac{\partial v_{\mathrm{a}}}{\partial p}-\frac{1}{2} \frac{\partial v_{\mathrm{r}}}{\partial p}\left(\frac{Y}{X}\right)^{2} \\
& -\frac{Y}{X} \frac{d\left(\frac{Y}{X}\right)}{d t} \frac{\partial X}{\partial p}
\end{aligned}
$$

Furthermore, assuming $X \gg Y$, we obtain

$$
\frac{\mathrm{d}}{\mathrm{d} t}\left(\frac{\partial r}{\partial p}\right) \simeq \frac{\partial v_{\mathrm{r}}}{\partial p}+\frac{\partial Y}{\partial p} \frac{v_{\mathrm{a}}}{X}+\frac{Y}{X} \frac{\partial v_{\mathrm{a}}}{\partial p}-\frac{1}{2} \frac{\partial v_{\mathrm{r}}}{\partial p}\left(\frac{Y}{X}\right)^{2}
$$

and

$$
\frac{\mathrm{d}^{2}}{\mathrm{~d} t^{2}} \frac{\partial r}{\partial p} \simeq \frac{\partial \dot{v}_{\mathrm{r}}}{\partial p}+\frac{\partial v_{\mathrm{a}}}{\partial p} \frac{v_{\mathrm{a}}}{X}+\frac{\partial Y}{\partial p} \frac{\dot{v}_{\mathrm{a}}}{X}+\frac{v_{\mathrm{a}}}{X} \frac{\partial v_{\mathrm{a}}}{\partial p}+\frac{Y}{X} \frac{\partial \dot{v}_{\mathrm{a}}}{\partial p}
$$

Proof: For the first part, it is a simple application of differential calculus. For the second part, we just have to remark that

$$
\begin{array}{ll}
\frac{\mathrm{d}\left(Y / X^{2}\right)}{\mathrm{d} t}=\frac{v_{\mathrm{a}}}{X^{2}}-2 v_{\mathrm{r}} \frac{Y}{X^{3}} \simeq 0 & \frac{\mathrm{d}(Y / X)}{\mathrm{d} t}=\frac{v_{\mathrm{a}}}{X}-Y \frac{v_{\mathrm{r}}}{X^{2}} \simeq \frac{v_{\mathrm{a}}}{X} \\
\frac{1}{X} \frac{\mathrm{d}(Y / X)}{\mathrm{d} t}=\frac{v_{\mathrm{a}}}{X^{2}}-Y \frac{v_{\mathrm{r}}}{X^{3}} \simeq 0 & \frac{\mathrm{d}(1 / X)}{\mathrm{d} t}=-\frac{v_{\mathrm{r}}}{X^{2}} \simeq 0
\end{array}
$$

\section{Appendix B: obtaining closed-form approximations of the $\Omega$ matrix}

Here is the proof of the Proposition 2 presented in Section 3 . Considering the approximation given by (7), it is convenient to define the following quantities

$$
X=r\left(t_{0}\right)+\int_{t_{0}}^{t} v_{\mathrm{r}}(\tau) \mathrm{d} \tau \quad Y=\int_{t_{0}}^{t} v_{\mathrm{a}}(\tau) \mathrm{d} \tau
$$

We want to evaluate the FIM for range observations and estimation of the parameter vector $\left(r, v, \theta_{t_{0}}, \beta\right)$.

Let us now derive convenient approximations of the $\boldsymbol{\Omega}_{[i, j]}$ coefficients:

1) $\boldsymbol{\Omega}_{[1,2]}$ :

Invoking (46) (Lemma 1) and (15), we obtain

$$
\boldsymbol{\Omega}_{[1,2]} \equiv \frac{\mathrm{d}}{\mathrm{d} t} \frac{\partial r}{\partial r\left(t_{0}\right)} \simeq \frac{\partial Y}{\partial r\left(t_{0}\right)} \frac{v_{\mathrm{a}}}{X}
$$

Now, we have

$$
\frac{\partial Y}{\partial r\left(t_{0}\right)}(t)=\int_{t_{0}}^{t} \frac{\partial v_{\mathrm{a}}(\tau)}{\partial r\left(t_{0}\right)} \mathrm{d} \tau=\int_{t_{0}}^{t} \epsilon(\tau) \mathrm{d} \tau
$$

Hence, $\partial Y /\left(\partial r\left(t_{0}\right)\right)\left[v_{\mathrm{a}} / X\right] \simeq 0$ and, finally, $\boldsymbol{\Omega}_{[1,2]} \simeq 0$.

2) $-\boldsymbol{\Omega}_{[1,3]}:$

Using (47) (Lemma 1) and approximation (17), we have

$$
\boldsymbol{\Omega}_{[1,3]} \equiv \frac{\mathrm{d}^{2}}{\mathrm{~d} t^{2}} \frac{\partial r}{\partial r\left(t_{0}\right)} \simeq \frac{\partial Y}{\partial r\left(t_{0}\right)} \frac{\dot{v}_{\mathrm{a}}}{X}
$$

and $\boldsymbol{\Omega}_{[1,3]} \simeq 0$.

3) $\bullet \boldsymbol{\Omega}_{[2,3]}$ :

$$
\boldsymbol{\Omega}_{[2,3]} \equiv \frac{\mathrm{d}^{2}}{\mathrm{~d} t^{2}} \frac{\partial r}{\partial v\left[y\left(t_{0}\right)\right]} \simeq \frac{\partial Y}{\partial v\left[y\left(t_{0}\right)\right]} \frac{\dot{v}_{\mathrm{a}}}{X}
$$

But, we have also

$$
\begin{aligned}
\frac{\partial Y}{\partial v\left[y\left(t_{0}\right)\right]}(t) & =\int_{t_{0}}^{t} \frac{\partial v_{\mathrm{a}}(\tau)}{\partial v\left[y\left(t_{0}\right)\right]} \mathrm{d} \tau \\
& \simeq \sin (\zeta) \int_{t_{0}}^{t} \frac{v(\tau)}{v\left(t_{0}\right)} \mathrm{d} \tau \leq \sin (\zeta) \cdot\left(t-t_{0}\right)
\end{aligned}
$$

because $v\left(t_{0}\right) \geq v(t)$. Thus, $\partial Y /\left(\partial v\left[y\left(t_{0}\right)\right]\right)\left(\dot{v}_{\mathrm{a}} / X\right) \simeq 0$, and $\boldsymbol{\Omega}_{[2,3]} \simeq 0$

4) - Let us now compute the diagonal terms of the first block. By (45) and approximation (15), we obtain

$$
\begin{aligned}
\boldsymbol{\Omega}_{[1,1]} & =\frac{\partial r}{\partial r\left(t_{0}\right)} \\
& \simeq 1+\int_{t_{0}}^{t} \epsilon(\tau) \cos (\zeta) \mathrm{d} \tau+\int_{t_{0}}^{t} \epsilon(\tau) \sin (\zeta) \frac{Y}{X} \mathrm{~d} \tau
\end{aligned}
$$

Similarly, (16) and (46) yield

$$
\boldsymbol{\Omega}_{[2,2]}=\frac{\mathrm{d}}{\mathrm{d} t} \frac{\partial r}{\partial v\left[y\left(t_{0}\right)\right]} \simeq \frac{v_{\mathrm{r}}}{v\left[y\left(t_{0}\right)\right]}+\frac{Y}{X} \frac{v_{\mathrm{a}}}{v\left[y\left(t_{0}\right)\right]}
$$

5) $\bullet \boldsymbol{\Omega}_{[4,3]}$ :

Equations (47) and (19) yield

$$
\begin{aligned}
\boldsymbol{\Omega}_{[4,3]}= & \frac{\mathrm{d}^{2}}{\mathrm{~d} t^{2}} \frac{\partial r}{\partial \beta} \simeq \frac{\partial \dot{v}_{\mathrm{r}}}{\partial \beta}+\frac{Y}{X} \frac{\partial \dot{v}_{\mathrm{a}}}{\partial \beta}+2 \frac{\partial v_{\mathrm{a}}}{\partial \beta} \frac{v_{\mathrm{a}}}{X}+\frac{\partial Y}{\partial \beta} \frac{\dot{v}_{\mathrm{a}}}{X} \\
\simeq & \frac{\dot{v}_{\mathrm{r}}}{\beta}+\left(\theta-\theta\left(t_{0}\right)\right) \frac{\dot{v}_{\mathrm{a}}}{\beta}+2 \frac{\left[v_{\mathrm{a}}(t)-v_{\mathrm{a}}\left(t_{0}\right)\right]}{\beta} \dot{\theta} \\
& +\frac{\left[Y(t)-Y\left(t_{0}\right)\right]-\left(t-t_{0}\right) v_{\mathrm{a}}\left(t_{0}\right)}{\beta} \ddot{\theta}
\end{aligned}
$$

The first part of the above approximation of $\boldsymbol{\Omega}_{[4,3]}$ is the ratio of the radial acceleration by $\beta$, and the second part can be $Y / X \simeq \theta, v_{\mathrm{a}} / X \simeq \dot{\theta}, \dot{v}_{\mathrm{a}} / X \simeq \ddot{\theta}$ (because $X$ is supposed much bigger than $Y$ ). Hence, the information about non-radial component is proportional to the variation of $\theta$. 6) - The $\theta$ terms:

To investigate the $\Omega$ coefficients related to the angle (i.e. partial derivatives w.r.t. $\theta$ ) $\theta$, we just have to compute two terms (because of the zeros in the matrix): $\boldsymbol{\Omega}_{[3,3]}$ and $\boldsymbol{\Omega}_{[3,4]}$. To do this, it is worth considering the relation between the range variation and the angular variation, as illustrated in Fig. 2, which is given by the following equations

$$
\sin (\tilde{\theta}(t))=\sin (\xi) \frac{\left\|\mathbf{r}(t)-\mathbf{r}\left(t_{0}\right)\right\|}{\|\mathbf{r}(t)\|}
$$

or

$$
\begin{aligned}
\sin (\tilde{\theta}(t))^{2}= & \sin (\xi)^{2} \\
& \times \frac{\|\mathbf{r}(t)\|^{2}+\left\|\mathbf{r}\left(t_{0}\right)\right\|^{2}-2\|\mathbf{r}(t)\|\left\|\mathbf{r}\left(t_{0}\right)\right\| \cos (\tilde{\theta}(t))}{\|\mathbf{r}(t)\|^{2}}
\end{aligned}
$$

Hence, since $\tilde{\theta}(t)$ is supposed to be small, using the 
second-order approximation of sine and cosine we deduce

$$
\begin{aligned}
r(t) & \simeq \frac{r\left(t_{0}\right)}{1-(\tilde{\theta}(t) / \tan (\xi))} \\
& \stackrel{2}{\simeq} r\left(t_{0}\right)\left(1+\frac{\tilde{\theta}(t)}{\tan (\xi)}+\frac{\tilde{\theta}(t)^{2}}{(\tan \xi)^{2}}\right)
\end{aligned}
$$

This allows us to obtain a simple expression of the derivative of the range against the initial angle

$$
\boldsymbol{\Omega}_{[3,1]}=\frac{\partial r}{\partial \theta\left(t_{0}\right)}=\frac{r\left(t_{0}\right)}{\tan (\xi)}-2 r\left(t_{0}\right) \frac{\tilde{\theta}(t)}{\tan (\xi)^{2}}
$$

and its successive derivatives, using $Y / X \simeq \theta, v_{a} / X \simeq \dot{\theta}$, $\dot{\theta}_{a} / X \simeq \ddot{\theta}$ (because $X$ is supposed much bigger than $Y$ )

$$
\begin{aligned}
\left(\boldsymbol{\Omega}_{[3,2]}\right) & =\frac{\partial \dot{r}}{\partial \theta\left(t_{0}\right)} \simeq \frac{-2 r\left(t_{0}\right) v_{\mathrm{a}}}{X \tan ^{2}(\xi)} \\
& =\frac{-2 r\left(t_{0}\right) v \cos ^{2}(\xi)}{X \sin (\xi)} \simeq \frac{-2 r\left(t_{0}\right) v \cos ^{2}(\xi)}{r(t) \sin (\xi)} \\
\left(\boldsymbol{\Omega}_{[3,3]}\right) & =\frac{\partial \ddot{r}}{\partial \theta\left(t_{0}\right)} \simeq \frac{-2 r\left(t_{0}\right) \dot{v}_{\mathrm{a}}}{X \tan ^{2}(\xi)} \\
& =\frac{-2 r\left(t_{0}\right) \dot{v} \cos ^{2}(\xi)}{X \sin (\xi)} \simeq \frac{-2 r\left(t_{0}\right) \dot{v} \cos ^{2}(\xi)}{r(t) \sin (\xi)} \\
\left(\boldsymbol{\Omega}_{[3,4]}\right) & =\frac{\mathrm{d}}{\mathrm{d} t} \frac{\partial \ddot{r}}{\partial \theta\left(t_{0}\right)} \simeq \frac{-2 r\left(t_{0}\right) \ddot{v} \cos ^{2}(\xi)}{r(t) \sin (\xi)}+\frac{2 r\left(t_{0}\right) \dot{r} \dot{v} \cos ^{2}(\xi)}{r(t)^{2} \sin (\xi)}
\end{aligned}
$$

7) $-\operatorname{det}(\boldsymbol{\Omega})$ :

Using these results, we can see that the matrix has the following structure

$$
\boldsymbol{\Omega}=\left(\begin{array}{cccc}
\boldsymbol{\Omega}_{[1,1]} & 0 & 0 & 0 \\
\boldsymbol{\Omega}_{[2,1]} & \boldsymbol{\Omega}_{[2,2]} & 0 & 0 \\
\boldsymbol{\Omega}_{[3,1]} & \boldsymbol{\Omega}_{[3,2]} & \boldsymbol{\Omega}_{[3,3]} & \boldsymbol{\Omega}_{[3,4]} \\
\boldsymbol{\Omega}_{[4,1]} & \boldsymbol{\Omega}_{[4,2]} & \boldsymbol{\Omega}_{[4,3]} & \boldsymbol{\Omega}_{[4,4]}
\end{array}\right)
$$

This leads to the general formula of the determinant. The evaluation itself is obtained by neglecting the term $\left.\left(2\left[v_{\mathrm{a}}(t)-v_{\mathrm{a}}\left(t_{0}\right)\right] / \beta\right) \dot{\theta}+\left(\left[Y(t)-Y\left(t_{0}\right)\right]-\left(t-t_{0}\right) v_{a}\left(t_{0}\right)\right] / \beta\right) \ddot{\theta}$ In $\boldsymbol{\Omega}_{[4,3]}$. Indeed in that case

$$
\boldsymbol{\Omega}_{[4,4]}=\frac{\mathrm{d}}{\mathrm{d} t} \boldsymbol{\Omega}_{[4,3]} \simeq \frac{\ddot{v}_{\mathrm{r}}}{\beta}+\tilde{\theta} \frac{\ddot{v}_{\mathrm{a}}}{\beta}+\dot{\theta} \frac{\dot{v}_{\mathrm{a}}}{\beta}
$$

Finally, collecting all the previous results, and the following (kinematic identities)

$$
\begin{array}{cl}
v_{\mathrm{r}}=v \cos (\zeta)=-v \cos (\xi) & v_{\mathrm{a}}=v \sin (\zeta)=v \sin (\xi) \\
\dot{v}_{\mathrm{r}} \ddot{v}=\ddot{v}_{\mathrm{r}} & \dot{v}_{\mathrm{a}} \ddot{v}=\ddot{v}_{\mathrm{a}}
\end{array}
$$

we have

$$
\begin{aligned}
& \boldsymbol{\Omega}_{[3,3]} \boldsymbol{\Omega}_{[4,4]}-\boldsymbol{\Omega}_{[4,3]} \boldsymbol{\Omega}_{[3,4]}=\frac{2 r\left(t_{0}\right) \cos ^{2}(\xi)}{r \sin (\xi)} \\
& \times\left\{-\dot{v} \frac{\ddot{v}_{\mathrm{r}}}{\beta}-\dot{\theta} \frac{\dot{v}_{\mathrm{a}} \dot{v}}{\beta}-\tilde{\theta} \frac{\ddot{v}_{\mathrm{a}} \dot{v}}{\beta}-\left(\frac{\dot{v}_{\mathrm{r}}}{\beta}+\tilde{\theta} \frac{\dot{v}_{\mathrm{a}}}{\beta}\right)\left(-\ddot{v}+\frac{\dot{r} \dot{v}}{r}\right)\right\} \\
& =\frac{2 r\left(t_{0}\right) \cos ^{2}(\xi)}{r} \frac{\dot{v}^{2}}{\beta}\left\{\frac{\dot{r}}{r} \tilde{\theta}-\dot{\theta}-\frac{\dot{r}}{r} \cot (\xi)\right\}
\end{aligned}
$$

This ends the proof. As a short endnote, we see that the terms are homogeneous; (66) is zero iff $\theta$ and $r$ are that is linearly dependent over time, that is $\mathrm{d} / \mathrm{d} t[\theta+\cot (\zeta)-$ $\left.\theta\left(t_{0}\right) / r\right]=0$.

\section{Appendix C: FIM determinant expansion}

We shall now turn towards expansions of the FIM determinant. Results are summarised in the following lemma.

Lemma 2: (expansion of the FIM around $\mathbf{t}_{\mathbf{0}}$ )

Let $P(p, q, r)$ be the following homogeneous polynomial

$$
P(p, q, r)=\frac{q r^{2}}{2}+\frac{p q^{2}}{2}+\frac{p^{2} r}{2}-\frac{q^{2} r}{2}-\frac{p r^{2}}{2}-\frac{p^{2} q}{2}
$$

and $Q(p, q, r, s)$ be

$$
\begin{aligned}
Q(p, q, r, s)= & \frac{1}{12}\left(q s^{2} r^{3}-q r^{2} s^{3}+q^{2} r s^{3}-q^{2} s r^{3}\right. \\
& +q^{3} s r^{2}-q^{3} s^{2} r+p r^{2} s^{3}-p s^{2} r^{3} \\
& +p q^{2} r^{3}-p q^{2} s^{3}+p q^{3} s^{2}-p q^{3} r^{2} \\
& +p^{2} s r^{3}-p^{2} s^{3} r+p^{2} q s^{3}-p^{2} q r^{3} \\
& +p^{2} q^{3} r-p^{2} q^{3} s+p^{3} s r^{2}-p^{3} s^{2} r \\
& \left.+p^{3} q s^{2}-p^{3} q r^{2}+q^{2} p^{3} s-q^{2} p^{3} r\right)
\end{aligned}
$$

Then, denoting $\tilde{t}_{i}=t_{i}-t_{0}$ the relative time differences, the following results hold:

- Consider the following second-order expansion of the gradient vector $\boldsymbol{M}_{\boldsymbol{t}}$ (see [5]):

$$
\boldsymbol{M}_{t_{i}} \stackrel{2}{=} \boldsymbol{M}_{t_{0}}+\tilde{t}_{i} \boldsymbol{M}_{t_{0}}^{(1)}+\frac{\tilde{t}_{i}^{2}}{2} \cdot \boldsymbol{M}_{t_{0}}^{2},
$$

$$
\begin{aligned}
\text { then } \operatorname{det}\left(\boldsymbol{M}_{t_{p}}, \boldsymbol{M}_{t_{q}}, \boldsymbol{M}_{t_{r}}\right)= & P\left(\tilde{t}_{p}, \tilde{t}_{q}, \tilde{t}_{r}\right) \operatorname{det} \\
& \times\left(\boldsymbol{M}_{t_{0}}, \boldsymbol{M}_{t_{0}}^{(1)}, \boldsymbol{M}_{t_{0}}^{(2)}\right)
\end{aligned}
$$

- Consider the following third-order expansion of the gradient vector $M_{t_{i}}$ (see [5]), then

$$
\begin{aligned}
\operatorname{det}\left(\boldsymbol{M}_{t_{p}}, \boldsymbol{M}_{t_{q}}, \boldsymbol{M}_{t_{r}}, \boldsymbol{M}_{t_{s}}\right)= & Q\left(\tilde{t}_{p}, \tilde{t}_{q}, \tilde{t}_{r}, \tilde{t}_{s}\right) \operatorname{det} \\
& \times\left(\boldsymbol{M}_{t_{0}}, \boldsymbol{M}_{t_{0}}^{(1)}, \boldsymbol{M}_{t_{0}}^{(2)}, \boldsymbol{M}_{t_{0}}^{(3)}\right)
\end{aligned}
$$

Proof: Let us denote $\tilde{t}_{i}=t_{i}-t_{0}$. Using the second-order expansion of the three-dimensional gradient vector $\boldsymbol{M}_{\mathrm{t}}$ and the fact that the determinant is multilinear and alternate, we obtain

$$
\begin{aligned}
& \left(\operatorname{det}\left(\boldsymbol{M}_{t_{p}}, \boldsymbol{M}_{t_{q}}, \boldsymbol{M}_{t_{r}}\right)\right. \\
& =\left(\frac{\tilde{t}_{q} \tilde{t}_{r}^{2}}{2}-\frac{\tilde{t}_{q}^{2} \tilde{t}_{r}}{2}-\frac{\tilde{t}_{p} \tilde{t}_{r}^{2}}{2}+\frac{\tilde{t}_{p} \tilde{t}_{q}^{2}}{2}+\frac{\tilde{t}_{p}^{2} \tilde{t}_{r}}{2}-\frac{\tilde{t}_{p}^{2} \tilde{t}_{q}}{2}\right) \\
& \quad \times \operatorname{det}\left(\boldsymbol{M}_{t_{0}}, \boldsymbol{M}_{t_{0}}^{(1)}, \boldsymbol{M}_{t_{0}}^{(2)}\right) \\
& =P\left(\tilde{t}_{p}, \tilde{t}_{q}, \tilde{t}_{r}\right) \operatorname{det}\left(\boldsymbol{M}_{t_{0}}, \boldsymbol{M}_{t_{0}}^{(1)}, \boldsymbol{M}_{t_{0}}^{(2)}\right)
\end{aligned}
$$


Quite similarly, for four-dimensional vectors and a third-order expansion of the three-dimensional gradient vector $\boldsymbol{M}_{t}$, we obtain

$$
\begin{aligned}
& 12 \operatorname{det}\left(\boldsymbol{M}_{t_{p}}, \boldsymbol{M}_{t_{q}}, \boldsymbol{M}_{t_{r}}, \boldsymbol{M}_{t_{s}}\right) \\
& =\left[\left(\tilde{t}_{q} \tilde{z}_{s}^{2} \tilde{t}_{r}^{3}-\tilde{t}_{q} \tilde{t}_{r}^{2} \tilde{t}_{s}^{3}\right)-\left(\tilde{t}_{q}^{2} \tilde{t}_{s} \tilde{t}_{r}-\tilde{t}_{q}^{2} \tilde{t}_{r} \tilde{t}_{s}^{3}\right)+\left(\tilde{t}_{q}^{3} \tilde{t}_{s} \tilde{t}_{r}^{2}-\tilde{t}_{q}^{3} \tilde{z}_{s} \tilde{t}_{r}\right)\right. \\
& -\left(\tilde{t}_{p} \tilde{t}_{s}^{2} \tilde{t}_{r}^{3}-\tilde{t}_{p} \tilde{t}_{r}^{2} \tilde{t}_{s}^{3}\right)+\left(\tilde{t}_{p} \tilde{t}_{q}^{2} \tilde{t}_{r}^{3}-\tilde{t}_{p} \tilde{t}_{q}^{2} \tilde{t}_{s}^{3}\right)-\left(\tilde{t}_{p} \tilde{t}_{q}^{3} \tilde{t}_{r}^{2}-\tilde{t}_{p} \tilde{t}_{q}^{3} \tilde{t}_{s}^{2}\right) \\
& -\left(\tilde{t}_{p} \tilde{t}_{s} \tilde{t}_{r}^{3}-\tilde{t}_{p}^{2} \tilde{t}_{s} \tilde{t}_{r}\right)-\left(\tilde{t}_{p}^{2} \tilde{t}_{q} \tilde{t}_{r}^{3}-\tilde{t}_{p} \tilde{t}_{q} \tilde{t}_{s}^{3}\right)+\left(\tilde{t}_{p}^{2} \tilde{t}_{q} \tilde{t}_{r}-\tilde{t}_{p}^{2} \tilde{t}_{q} \tilde{t}_{s}\right) \\
& \left.-\left(\tilde{t}_{p}^{3} \tilde{t}_{s} \tilde{t}_{r}^{2}-\tilde{t}_{p}^{3} \tilde{t}_{s} \tilde{t}_{r}\right)+\left(\tilde{t}_{p}^{3} \tilde{t}_{q} \tilde{t}_{r}^{2}-\tilde{t}_{p}^{3} \tilde{t}_{q} \tilde{t}_{s}^{2}\right)-\left(\tilde{t}_{q}^{2} \tilde{t}_{p} \tilde{t}_{r}-\tilde{t}_{q}^{2} \tilde{t}_{p} \tilde{t}_{s}\right)\right]
\end{aligned}
$$

$$
\begin{aligned}
& \times \operatorname{det}\left(\boldsymbol{M}_{t_{0}}, \boldsymbol{M}_{t_{0}}^{(1)}, \boldsymbol{M}_{t_{0}}^{(2)}, \boldsymbol{M}_{t_{0}}^{(3)}\right) \\
= & Q\left(\tilde{t}_{p}, \tilde{t}_{q}, \tilde{t}_{r}, \tilde{t}_{s}\right) \operatorname{det}\left(\boldsymbol{M}_{t_{0}}, \boldsymbol{M}_{t_{0}}^{(1)}, \boldsymbol{M}_{t_{0}}^{(2)}, \boldsymbol{M}_{t_{0}}^{(3)}\right)
\end{aligned}
$$

Lemma 3: (some useful identities)

$$
\begin{aligned}
& \sum_{1 \leq p \leq q \leq k}\left(q-p^{2}\right)=\frac{1}{12} k^{2}(k-1)(k+1) \\
& \sum_{1 \leq p \leq q \leq k}\left(p^{2} q-q^{2} p\right)^{2}= \frac{1}{720} k^{2}(k-1)(k+2) \\
& \times\left(3 k^{2}+3 k+2\right)(k+1)^{2}
\end{aligned}
$$

